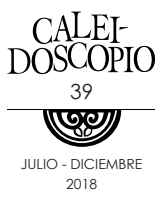

\title{
Binomios y dicotomías en las representaciones sociales de la maternidad en madres adolescentes
}

Pairs and dichotomies in the social representations of motherhood in adolescent mothers

BÉLGICA MARÍA ROMERO DE LOERA'

\section{RESUMEN}

Este artículo tiene como propósito comprender cómo es concebida la maternidad desde las mismas madres jóvenes. Se parte de la teoría de las representaciones sociales para dar cuenta de las concepciones construidas y reconstruidas por jóvenes menores de 20 años que son madres y relatan parte de sus vivencias, pensamientos y sentimientos en entrevistas a profundidad que fueron estudiadas bajo la técnica de análisis cualitativo de contenido, partiendo de categorías que emergieron tras el análisis de cartas asociativas. Los diferentes elementos de representación social encontrados están fuertemente asociados entre sí: (1) el apoyo social con los retos y exigencias, (2) la compensación con los retos y con los roles/relación, (3) las renuncias con el temor/sufrimiento, y (4) el temor/sufrimiento con procesos de corporalidad. Estas representaciones se organizan en pares de opuestos: las exigencias frente a las renuncias, la compensación frente al temor/sufrimiento y la compensación frente a la trascendencia. Las representaciones sociales tienen como centro el apoyo social, las exigencias y las renuncias, las cuales dan significado a la maternidad de las jóvenes y permiten hacer inteligible la información del contexto, orientar sus acciones y comunicarse en los escenarios sociales en los cuales se desenvuelven.

Palabras clave: representaciones sociales, maternidad, jóvenes, sentido común, análisis de contenido.

1 Universidad Cuauhtémoc Aguascalientes, México. 
This article aims to understand how premature motherhood is conceived by young mothers. Based on Social Representation Theory, the study seeks to make sense of how those conceptions are constructed and reconstructed by young mothers under 20, who shared their experiences, thoughts, and feelings through in-depth interviews. Interview transcripts were submitted to qualitative content analysis guided by categories from a previous study conducted through associative cards. Resulting social representation components are highly linked among them: (1) social support with challenges and demands, (2) compensation with challenges and roles/relationship, (3) resignation with fear/ suffering, and (4) fear/suffering with transcendence. These representations are organized in pairs of opposites: demands vs. resignation, compensation $v$ s. fear/suffering, compensation vs. transcendence. Social support, demands and resignation are at the center of the social representations. They allow young women for the signification of maternity so that they can make sense of information regarding the context, focus their actions and communicate in their social environments.

Keywords: social representations, materhood, youth, common sense, content analysis.

En la sociedad occidental actual, ser joven conlleva representaciones sobre los modos correctos de ser y estar que no dan cabida a la maternidad. Por ello, además de las dificultades que implican el cuidado y crianza de los hijos, las madres adolescentes deben enfrentar la discriminación derivada de encontrarse en una situación indeseable o proscrita.

El presente artículo recoge los resultados de una investigación que tuvo como finalidad comprender los contenidos de las representaciones sociales de la maternidad que comparten madres jóvenes de un contexto urbano en una ciudad media. Las preguntas que guiaron esta investigación de corte cualitativo fueron: ¿cuáles son los elementos de constitución de las representaciones sociales de la maternidad temprana en madres menores de 20 años?, ¿qué relación existe entre estos elementos?, y ¿cuál es la funcionalidad de las representaciones sociales de la maternidad temprana para las jóvenes de manera individual y para su contexto? 
La investigación tiene como antecedente una serie de estudios que demandan alejarse de la visión del problema de salud pública con el que se ha estudiado el fenómeno en aras de su prevención, otorgándole, por otra parte, un carácter social (Climent, 2003; 2009; Climent, Arias y Spurio, 2000; Llanes, 2014; Ortiz y Maza, 2010; Román, 2000; Stern, 2012). Climent (2003) considera que al plantearse la maternidad adolescente como un problema social es necesario preguntarse si es una pauta cultural que debe respetarse o si, por el contrario, es un evento de consecuencias indeseables para la vida de las mujeres y sus familias. En todo caso, sería necesario ahondar en las funciones que la maternidad estaría teniendo en la vida de estas jóvenes, las carencias que estaría cubriendo.

Ya de manera específica, desde la aproximación teórica que orienta esta investigación, surge la pregunta sobre el modo en que ha sido abordado el tema de las representaciones sociales de la maternidad. Distintos estudios revelan la centralidad de la maternidad dentro de las representaciones sociales de las mujeres, ya sea mediante el análisis de producciones sociales, tales como el arte, (Álvaro y Fernández, 2006) o del discurso de las propias mujeres (Schwarz, 2005). Las representaciones sociales que tienen las madres jóvenes han sido analizadas recientemente en dos investigaciones para nosotros paradigmáticas, la de Lamus (1999) en Colombia y la de Climent (2009) en Argentina. En dichos estudios aparecen: el nuevo estatus social, miedo al rechazo familiar, decisiones trascendentales, postergación de estudios, padre del bebé como proveedor, y dependencia familiar y económica como contenidos de la representación social de la maternidad en las jóvenes. En el caso de las madres multigestantes, aparecen además el padre del hijo como contenedor emocional y el bebé como fuente de amor. En la investigación de Climent (2009), la polaridad de los elementos determina si en las representaciones sociales de las jóvenes y en las hegemónicas el embarazo y la maternidad adolescentes son vistos como problema o no. En muchos de los casos la maternidad "es vista como una búsqueda de afirmación social y afecto, una forma de realización personal que incluso eleva la autoestima, presentando así una connotación positiva del embarazo" (Lamus, 1999: 196). Ello convierte a la maternidad de estas jóvenes en parte central de su proyecto de vida y, en algunas ocasiones, en la única función de la mujer.

A partir de esta revisión, la investigación tuvo como objetivo general comprender la base de la constitución y funcionalidad de las

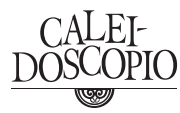


representaciones sociales de la maternidad temprana en madres menores de 20 años. Se trata de conocer los contenidos de dichas representaciones sociales y la relación entre estos contenidos con el fin de analizar la funcionalidad que tienen estas representaciones para las madres jóvenes y para la cultura en la cual están inmersas.

\section{TEORÍA DE LAS REPRESENTACIONES SOCIALES}

En un sentido concreto, la teoría de las representaciones sociales es una teoría acerca del sentido común, por lo cual consideramos pertinente iniciar el acercamiento a la teoría identificando las características del sentido común al que nos referiremos. El pensamiento de sentido común está fuertemente influido por las creencias anteriores o los estereotipos del lenguaje (Moscovici y Hewstone, 1986); se constituye a partir de las experiencias, pero también de la información, conocimientos y modelos de pensamiento que recibimos. Es un conocimiento práctico socialmente elaborado y compartido (Jodelet, 1985), y se diferencia de otras producciones sociales como la ciencia, el mito, la religión o la ideología por sus formas de elaboración y funcionamiento. Son precisamente esas reglas de elaboración, funcionamiento y, nosotros agregamos, de práctica en la vida cotidiana, el foco de interés de la teoría de las representaciones sociales.

El trabajo de Moscovici, que inicia la teoría de las representaciones sociales, otorga una densidad propia al sentido común o conocimiento cotidiano producido en las interacciones sociales, e identifica que las representaciones sociales que lo conforman no están limitadas por las reglas del discurso lógico ni por los procesos de verificación empírica (Castorina, 2003). La teoría de las representaciones sociales considera al agente social (individual o grupal) como productor de sentidos que construye realidades.

Sin omitir la dirección contraria en el binomio estructura-agente, esta teoría pone el foco en las producciones simbólicas, los significados y el lenguaje, a través del cual se produce el mundo. Este foco que atiende a la necesidad de explicar la conformación del individuo social $\mathrm{y}$, al mismo tiempo, de la sociedad, es una de las razones del creciente interés por el uso de esta teoría en investigaciones empíricas. El concepto de representación social es el de una modalidad de pensamiento social, ya que se asume que es un conocimiento socialmente 
constituido que se pone en marcha en las interacciones sociales y a partir de fenómenos sociales; de la realidad social. Dicho conocimiento está conformado por componentes cognoscitivos y emocionales (información y actitud), organizados dentro de una lógica interna y compartida por los miembros de una cultura.

A partir de la conceptualización y el estudio empírico de Moscovici y sus seguidores -las escuelas de Jodelet, Abric y Doise-, se tienen identificadas tres dimensiones de las representaciones sociales y dos procesos básicos. Las tres dimensiones son relativas al contenido:

Actitud. Es la dimensión afectiva de la representación, le imprime un carácter dinámico y orienta el comportamiento hacia el objeto de la misma; dotándolo de reacciones emocionales de diversa intensidad y dirección (Perera, 1999). Esta estructura permite dirigir no solamente las conductas, sino la orientación y selección de la información a la cual estamos expuestos.

Información. Esta dimensión expresa los conocimientos en torno a un objeto de representación. El acceso al conocimiento que circula en una cultura está mediado por la pertenencia a un grupo y la posición social de los individuos respecto al grupo.

Muchas de las investigaciones en torno a las representaciones sociales se focalizan primordialmente en conocer ese cuerpo de conocimientos y de actitudes. Sin embargo, la riqueza de la teoría se da cuando se identifican las relaciones y organizaciones entre ambos elementos, lo cual corresponde a un tercer elemento: el campo de representación.

Campo de representación. Esta dimensión se refiere a la organización que toman los contenidos representacionales, siendo éstos en torno a un núcleo con elementos centrales y periféricos. La relación entre los elementos y su jerarquía se establece como modelo figurativo durante el proceso de objetivación. Está compuesta por cogniciones y actitudes que dotan de significación al resto de los elementos. Esta dimensión es construida teóricamente por el investigador al momento de analizar cada uno de los elementos, como lo son actitudes, opiniones, creencias, vivencias, valores en torno a un mismo objeto de representación. Este elemento nos da cuenta de la estructura de la representación social.

En conclusión, para conocer y explicar una representación social es necesario determinar lo que la persona o grupo conoce (información), cómo se interpreta o se constituye como creencia (campo de repre-

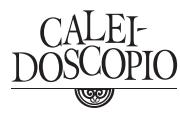


sentación) y cómo se actúa o qué se hace con estas interpretaciones (actitud). Para ello, analizar el proceso de la construcción y puesta en marcha de una representación social implica abordar los procesos básicos de objetivación y anclaje, ya que éstos explican cómo lo social transforma un conocimiento en representación y cómo esta representación transforma lo social.

Las representaciones sociales median la relación entre dos elementos esenciales: el objeto entendido como fenómeno social o realidad social y el sujeto entendido como individuo o como grupo. Ambos, objeto y sujeto en una relación dialógica, se encuentran en un contexto social y cultural. El contexto provee información, mediando su acceso por la posición y pertenencia del sujeto. A través del proceso de objetivación, esta información permite incorporar lo nuevo al conocimiento preexistente y conforma la información sobre el objeto. El proceso de objetivación se da en tres momentos: (1) selección y descontextualización de la información; (2) formación del núcleo figurativo; y (3) naturalización. Una manera de poder identificar este proceso de objetivación es a través de las metáforas, ejemplos, imágenes y justificaciones acerca del objeto de la representación. Como contenidos de las representaciones sociales se ubican tanto esta información como las actitudes y el campo de representación.

Los contenidos están dinámicamente relacionados, teniendo así que las actitudes son la parte emotiva de la representación y cuya manifestación en relación con la información son los estereotipos. Lo que organiza ambos contenidos de la representación es el campo representacional. Este último contenido permite la comunicación entre las personas, la significación y el uso de la representación social que, a través del proceso de anclaje, inserta las representaciones en el contexto, construyendo la sociedad y siendo alimento de nuevas representaciones sociales.

\section{MÉTODO}

Además de proporcionar un marco conceptual para la explicación de fenómenos sociales, la teoría de las representaciones sociales ofrece una postura metodológica. Abric (2001) señala que, para la investigación centrada en representaciones sociales, las técnicas utilizadas pueden clasificarse como interrogativas y asociativas. Ambas se enfocan 
en recoger una expresión verbal, pero las segundas buscan aquellas expresiones más auténticas, espontáneas y, por ende, más inmediatas.

En esta investigación se utilizó como técnica interrogativa la entrevista individual a profundidad, para conocer el contenido de las representaciones sociales de las jóvenes respecto a la maternidad temprana, el origen de la información sobre ser madre que las jóvenes identifican en su contexto y las actitudes hacia esta información; así como la descripción que hacen las jóvenes sobre la vida cotidiana en la crianza de los hijos. Se realizaron cinco entrevistas a partir de una guía, en la cual se incluyeron los datos de identificación de las jóvenes, la firma de un consentimiento informado y la pregunta: ¿qué es para ti la maternidad temprana? A partir de esta pregunta generadora se incluyeron en la guía los temas de maternidad, hijos, proyecto de vida, escuela, trabajo, pareja, educación de los hijos, relación con los padres, historias de maternidades tempranas en la familia, cambios a partir de la maternidad y actividades de un día común como mujer, como madre y como algún otro rol (estudiante o trabajadora). Cada entrevista fue grabada en audio y transcrita para su análisis.

Como técnica asociativa se utilizó la carta asociativa, la cual permite acceder al campo semántico de la persona, conociendo el significado de una serie de términos nodales dentro de una red conformada por cadenas de asociación. Además de visualizar la representación social, la carta asociativa tiene la ventaja de que permite identificar elementos centrales y periféricos y, sobre todo, conocer el sentido que cada persona otorga a los contenidos. Por último, se destaca la ventaja de ser una técnica proyectiva que, como tal, permite acceder al mundo cognitivo e individual de cada persona con una menor conciencia o racionalidad que otras técnicas, debido a la inmediatez de respuesta que se promueve ante cada término inductor.

El trabajo de campo fue realizado entre febrero y noviembre de 2015. Las cartas asociativas y el levantamiento de datos socioeducativos fueron realizados mediante un mismo instrumento. Para esta investigación, las cartas asociativas tuvieron como término inductor -a partir del cual se generaron asociaciones libres- la palabra "maternidad". Se recogieron redes de tres niveles o cadenas de asociaciones para cada una de las 59 cartas aplicadas a igual número de madres jóvenes de 14 a 20 años de edad. Al concluir la aplicación de cada carta asociativa, se le preguntó a cada joven si deseaba participar en una

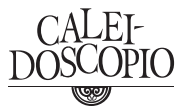


entrevista individual para la misma investigación y solamente a las que accedieron se les pidió proporcionar datos de contacto.

De las cinco jóvenes entrevistadas, dos (Ana y Mayra ${ }^{2}$ ) provienen de un estrato social medio, con padres profesionistas, divorciados y actualmente estudian la licenciatura de manera escolarizada. Otras dos jóvenes (Diana y Jessica) cuentan solamente con educación básica y no han continuado sus estudios; ellas provienen de hogares de extractos sociales pobres. Por su parte, Blanca, la quinta joven entrevistada, proviene de un hogar de estrato social pobre y actualmente se encuentra estudiando la preparatoria de manera abierta. Dos de las jóvenes (Ana y Blanca) viven con sus parejas, mientras que el resto (Mayra, Diana y Jessica) vive con sus padres. Se considera que las cinco jóvenes entrevistadas representan los principales estratos sociales y antecedentes educativos y familiares que permiten tener unidades de análisis de discursos y significaciones, que pueden estar presentes en los sectores de la población en los que se presenta con mayor incidencia el fenómeno de la maternidad temprana.

El análisis de la información se realizó a través del método de análisis de contenido para determinar las conexiones entre el nivel sintáctico del texto y sus niveles semántico y pragmático (Navarro y Díaz, 1995). El procedimiento general de la técnica incluyó de manera general los siguientes elementos:

1. Determinación del objeto o tema de análisis. Para esta investigación el tema de análisis son los significados o representaciones sociales de la maternidad temprana.

2. Determinación de las reglas de codificación. En primer lugar, las reglas de codificación se realizaron a partir de los criterios de presencia, frecuencia, orden y contingencia de los términos en las cartas asociativas. Posteriormente, se codificaron las categorías y fueron trasladadas como códigos analíticos a las entrevistas.

3. Determinación del sistema de categorías. Los ejes de análisis emergieron del estado de la cuestión y de forma empírica a partir de la red de asociaciones entre los términos de las cartas asociativas aplicadas a madres jóvenes.

4. Comprobación de la fiabilidad del sistema de codificación-categorización. La fiabilidad del sistema de codificación-categorización fue

2 En todos los casos se emplean pseudónimos para referirse a las madres entrevistadas. 
revisada a partir de la inclusión de una segunda persona como codificador, de manera que se discutió la concordancia entre el proceso y el resultado.

5. Inferencias. El proceso de interpretación y las inferencias surgidas se dan a partir del marco teórico. La significación de las representaciones sociales de la maternidad temprana se estableció a partir de los elementos de anclaje, objetivación y organización de los contenidos categorizados.

\section{RESULTADOS}

Las jóvenes que participaron en el estudio de cartas asociativas comparten la característica de ser madres de, al menos, un hijo. La mayoría (50 jóvenes) contaban con un hijo, 8 jóvenes con dos hijos y solamente una contaba con tres hijos al momento de la aplicación. Aunque en un primer momento se buscaba integrar solamente a jóvenes mayores de 16 años, por la cantidad de informantes a las que se tuvo acceso, y dado que las asociaciones surgidas en la carta asociativa eran similares al resto, se decidió integrar la respuesta de 6 jóvenes de 14 y 15 años, siendo la edad media de la muestra de 17.7 años. En relación a los antecedentes educativos de las jóvenes, la mayor parte cuenta con educación básica concluida (primaria y secundaria); solamente 15 de ellas tienen el bachillerato terminado. Más de la mitad de las informantes no se encontraba estudiando.

En cuanto al contexto familiar y económico, se identificó que gran parte de las madres jóvenes ( 46 de 59) viven con sus padres o con los de su pareja; sólo algunas de las jóvenes son mantenidas por sus familiares (23 de 59); el resto son mantenidas por sus parejas. En 12 de los casos, ellas hacen aportaciones a la manutención de los hijos. La edad promedio de los padres de las jóvenes es de 47.5 para el padre y 30.9 para la madre, mientras que la escolaridad de los padres es, en su mayoría, la educación básica concluida, tanto para padres como para madres. Nueve padres y ocho madres con estudios superiores y de posgrado son quienes tienen el nivel educativo más alto.

A partir de las redes de asociación que se desprendían de cada una de las palabras que las jóvenes mencionaron ante el término inductor "maternidad" en las cartas asociativas, se categorizaron todas las palabras de la primera red de asociación. Al momento de establecer

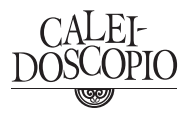


la categoría, se construyó la definición de la misma, considerando la concatenación de palabras que caracterizaba esa red de asociaciones. Con base en las entrevistas, la definición de cada categoría se hizo más amplia. El resultado final incluye las nueve categorías que se explican a continuación.

\section{EXIGENCIAS}

Definida como los requerimientos que demandan a la madre el cuidado y crianza de los hijos y ante los cuales ella responde, asumiendo la responsabilidad con un gasto energético, económico y emocional. La frecuencia de aparición de asociaciones con exigencias es de 293, siendo el concepto más frecuentemente asociado el de responsabilidad. La exigencia forma parte central del contenido de la representación social de la maternidad, como lo refieren las jóvenes entrevistadas en sus discursos. En cuatro de los casos, ante la pregunta expresa inicial en las entrevistas sobre el significado de la maternidad, el concepto que surge de inicio es la responsabilidad, que incluye preocupaciones y acciones de tipo económico, relacionadas con la crianza y con el tiempo que ello implica. Las actividades que más se detallan como indicador de la responsabilidad que significa la maternidad son las de la crianza de los hijos, que incluye no sólo los cuidados de alimentación e higiene, sino la educación. En los discursos de las jóvenes se ilustra cómo significan la maternidad a través de la interiorización de la responsabilidad: "[... y y ahora veo todo lo que implica, desde gastos, desde la educación del bebé, el tiempo que le dedicas, además te hace ser más responsable y todo [...] (Blanca, 16 años).

En todos los casos encontramos referencia a que es precisamente la maternidad la causa directa de esa responsabilidad y las jóvenes rápidamente se dan cuenta de ello, al interiorizar la responsabilidad como parte central del contenido de la representación social del contexto. Tal es el caso de Ana cuando nos comenta:

[...] mi esposo y yo desde un poquito antes, de hecho llevamos viviendo juntos cuatro meses, decidimos ya vivir solos, ¿por qué?, porque pues si estás... bueno así lo veo yo, si estás teniendo... si vas a tener una responsabilidad que es de un adulto ya mayor de 25 años, ponle, ya con una mentalidad más grande, con más preparación, pues te tienes que enfo- 
car en ese papel porque pues, ¿de qué sirve que me ponga a berrinchear de que "ay, estoy muy chiquita"? [imita sonidos de berrinche]. O sea no, no tiene sentido achicarme más de lo que estoy ahorita para cuidar a un niño, aniñarme más. Entonces prefiero... ambos preferimos madurar, hacernos independientes, hacernos responsables y empezar a vivir solos, a ver cómo nos iba y pues sí nos fue muy bien al principio, bueno, siempre ¿no? [risas] (Ana, 19 años).

La responsabilidad total de la maternidad que recae sobre la joven es parte esencial de la socialización de género que las mujeres han venido interiorizando desde pequeñas en el contexto cercano. En esta socialización, el papel de las madres de las jóvenes es importante en el momento de la maternidad. Son las madres las que recuerdan a las jóvenes que la responsabilidad es un aspecto central de la maternidad y que deben asumirla por completo.

Por otra parte, la responsabilidad como exigencia de la maternidad es vivida desde el compromiso, pero también desde el desgaste energético, económico y emocional que manifiestan las jóvenes; así, la exigencia se percibe en momentos como "difícil" o "cansada". El desgaste energético se manifiesta en la dificultad que les supone la organización del tiempo para cumplir los compromisos de la maternidad. De hecho, el concepto de tiempo y la reducción del mismo para cumplir con las actividades de la crianza de los hijos, en combinación con otros roles como la escuela o trabajo, es el que se encontró más asociado a la responsabilidad y a la maternidad en todas las entrevistas. Las jóvenes dan respuestas como las siguientes:

[Ante la pregunta de si ha encontrado dificultades] "[...] cambió totalmente mi vida. Ahora tengo que enfocarme a él y se me hace un poco difícil, pero ya me estoy acostumbrando" (Diana, 19 años).

[Ante la pregunta de cambios a partir de la maternidad] "Uy, pues mucho, el tiempo, el tiempo ha cambiado mucho. Me refiero a que de que antes no tenía que ponerme tiempos. O sea, a esta hora voy a hacer esto, ahora voy a hacer esto, etcétera, y ahorita sí... siempre estoy midiendo el tiempo" (Ana, 19 años).

Podemos concluir que las exigencias son un contenido central de la maternidad, y otorgan su significación de responsabilidad en concordancia con el contexto de las jóvenes, pero asumido con compromiso por parte de ellas, no sin reconocer el desgaste energético que conlleva.

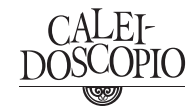




\section{APOYO SOCIAL}

Esta categoría está caracterizada por conceptos que aluden a la edad, roles y personas cercanas a la joven; con una frecuencia de 56, ha sido definida como la necesidad de apoyo social brindada por o demandada a la red social de apoyo de la joven.

En las entrevistas queda de manifiesto cómo las personas que brindan apoyo social, o aquellas de las que las jóvenes lo esperan, forman parte central de la representación social de maternidad. Es la familia quien suele cubrir la mayor parte de las necesidades de apoyo de las jóvenes. El apoyo social por parte de la familia puede ser característico de la maternidad temprana, un elemento para distinguir la significación de la maternidad en otras edades:

Pues más que nada uno se hace no tanto independiente, porque cuando uno es mamá soltera, dependes de tus papás porque... porque a... pues, ¿a quién más le pides apoyo?, ¿a quién más?, porque ellos son los que te apoyan, los hermanos, porque cuando no te apoya el hombre, ¿qué haces? Y ahora sí, como dicen, ya corres pues con la mamá, con el papá y pues tus papás son como uno. Yo nunca dejaría a m’hijo solo y mi papá y mi mamá y nuestros papás nunca nos van a dejar solas (Jessica, 16 años).

En cuatro de los casos, la necesidad de apoyo social es satisfecha principalmente por la madre de la joven:

[... la mujer siempre tiene que cuidar a su hijo y no va a tener ayuda de su esposo sino de su mamá y de la otra gente. El papá de los bebés no [...], y es que una mamá te ayuda más que un papá porque ella vio, sabió [sic], sintió lo que se siente y por eso ella: "iay, amá! es que el niño tiene esto" y "a ver, vamos", y un papá "ay no... es que, a ver con qué se lo quitas" que "ponle esto". Y la mamá, o sea la mamá siempre está apurada "a ver, amos [sic] a llevarlo al hospital" porque ellas supieron lo que es criar a un hijo (Jessica, 16 años).

“|...] pues a veces dice mi mamá 'no pues, aguántate, es tu hijo' [risas], y sí, claro que me ayuda" (Diana, 19 años)

Las jóvenes también identifican a la pareja como una persona de la que reciben o esperarían recibir apoyo social. En este caso, dos de ellas manifiestan la necesidad no satisfecha de apoyo por parte de su pareja 
(Jessica y Diana), mientras que tres de ellas sí reciben el apoyo, aun y cuando una de ellas (Mayra) no vive con su pareja. Ana y Blanca no sólo cuentan con el apoyo económico de los padres, sino que además viven con ellos y cuentan con su apoyo en las actividades de crianza.

\section{ROLES/RELACIÓN}

Con una frecuencia de aparición de 95 asociaciones entre mamá e hijo, la categoría se define como estos dos conceptos y sus variantes: bebé, madre, niño, entre otras, que solamente son posibles en relación y que no incluyen asociaciones ascendentes o descendentes más allá del término inductor "maternidad". Esta relación es vista por las jóvenes como única, íntima, cercana y de correspondencia mutua, donde la madre provee cuidados y amor, mientras recibe amor de parte del hijo. Esta relación se manifiesta de forma limitada en las cartas asociativas, dadas las limitaciones de la técnica para abordar de forma comprensiva las relaciones conceptuales que hacen las jóvenes. Esto difiere de lo que posibilitan las entrevistas, en las que la libertad de explicar cada una de las relaciones conceptuales hace que la asociación de la maternidad, como relación madre-hijo, pueda verse, por ejemplo, a través de la noción de instinto y que para Ana es el primer y central significado que se manifiesta de manera automática con el nacimiento de su hijo:

[Ante la pregunta del significado de la maternidad] Pues en este momento me siento como si fuera un instinto... Me sentía... |al momento de nacer su hijol Lo voy a decir de esta manera primitiva, no sé si me entiendas, así yo me sentía. Como si... como un simio, por decir así, me sentía como un animal. Sí, más animal de lo que me siento... me sentía anteriormente como una niña ¿no? mhhh, pero después cuando nació, ya como un instinto maternal que no se me hizo raro, como si anteriormente ya hubiera sido mamá de alguna manera... como me lo dieron lo supe cargar, no me ayudó nadie, no se me ha caído. Entonces me siento como... como ¡wow! Entonces si describiera la maternidad con una palabra sería instinto. [... ¿Qué es la maternidad? Yo te digo que es un instinto porque no estaba preparada (Ana, 19 años).

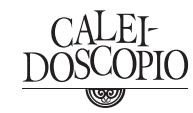


La relación madre-hijo es vista como una relación en donde ambas partes aportan algo importante para que la relación sea cercana, íntima, y la maternidad sea significada por esa relación de reciprocidad entre cuidados instrumentales y sentimientos positivos que la joven percibe como naturales y originados por su hijo.

\section{COMPENSACIÓN}

La frecuencia de esta categoría es de 149. En la categoría se incluyen sentimientos y adjetivos con carga emotiva considerada socialmente como positiva, tales como amor, cariño y que son referidos a la experiencia de la maternidad, la relación con el hijo y el nuevo rol. Las entrevistadas reconocen estos efectos positivos en sí mismas:

[Ante la pregunta de sentimientos asociados a la maternidad] "Podría decir emoción, felicidad... No sé por qué es una felicidad muy diferente, es una felicidad enlazada con emoción y con alegría. O sea, es como todas las emociones hermosas juntas en una sola, no sé cómo llamarlo, como emoción-amor-felicidad-alegría [risas]" (Ana, 19 años).

“[...] pues a veces también son un poquito de problemas, pero yo creo que el amor que una mamá siente, eso te impulsa, no importa el problema que sea, tú sales adelante por tu hijo, ¿verdad?" (Diana, 19 años).

"Bueno, para mí fue lo mejor que me pudo haber pasado. No sé, como que me cambió la vida. Bueno, porque yo antes era rebelde y así y ya no con mi niño, con mi hijo ya no" (Jessica, 16 años).

"[...] me doy cuenta de que es algo muy bonito y más que nada porque ya no nada más eres tú, ya tienes con quien compartir lo que sientes y con quien compartir momentos bonitos, para mí eso es la maternidad. [... Cambió mi forma de ver las cosas. Por ejemplo, ahora que estoy en la preparatoria veo muchos compañeros que no valoran el estudio" (Blanca, 16 años).

"Pues muchas cosas, como ahora que lo soy [madre], como que siento que soy una mejor persona, soy una persona mucho más humilde, mucho más centrada, yo creo que aunque a veces muchas personas digan, 'ay sí, vas a ser madura porque tuviste un hijo a los no sé cuántos años'. Pero la verdad es que no sé, sí agarras la onda" (Mayra, 19 años). 
Todas las entrevistadas identifican y manifiestan cambios positivos percibidos en sí mismas a partir de la maternidad, que se refieren a la autopercepción de madurez y humildad. Se consideran "mejor persona" y ven al hijo como una fuente de amor, compañía e impulso para afrontar las dificultades que puedan o se estén presentando en su vida. La compensación de la maternidad se ve reflejada, no sólo en sí mismas, sino que las jóvenes entrevistadas atribuyen cambios positivos en sus relaciones familiares a partir del nacimiento del hijo. La compensación también se manifiesta en modificaciones positivas en relación a la pareja, ya sea como en el caso de Ana, que refiere que ahora con su hijo su relación de pareja es "más íntima", o como Diana, a quien la llegada del hijo le permite dejar una relación de pareja violenta: "[...] creo que si no lo hubiera tenido [refiriéndose al hijo], tal vez seguiría con él [refiriéndose a su pareja]. Por mi hijo yo, pues él me dio fuerzas para decir '¿sabes qué?, ya, hasta aquí', entonces creo que fue algo muy bueno para mí" (Diana, 19 años).

En las cartas asociativas, la categoría de la compensación apareció frecuentemente asociada a la maternidad; justo por debajo de las exigencias. Ello parece indicar que las jóvenes representan socialmente la maternidad como una exigencia que al ser asociada a contenidos positivos o ganancias, permite convertir dichas exigencias en retos asumidos con un poco más que aceptación. En el caso de las entrevistas, podemos ver cómo esta relación entre exigencias y compensación se da cuando las jóvenes parece que renegarán de la responsabilidad de la maternidad, para ellas mismas autocensurarse con un sentimiento positivo que provee la maternidad. Es quizá debido a esta relación estrecha entre los contenidos de exigencias y compensaciones de la maternidad que las entrevistadas no hablaron de alternativas ante el embarazo; sólo la de asumir la responsabilidad de la maternidad. La excepción es el caso de Blanca, quien contó con tristeza y llanto que su madre le había sugerido abortar y que quizá tendría culpa por haberlo sugerido.

\section{RENUNCIAS}

Se refiere a todas aquellas actividades que están relacionadas con lo que se entiende como pérdida de libertad; actividades tales como fiestas, estudio o salidas. Los términos de esta categoría se presen-

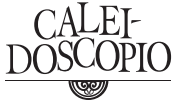


tan de manera frecuente (frecuencia de 20) en las cartas asociativas. Las jóvenes manifiestan melancolía o sentimiento de pérdida ante tales actividades. Ante las exigencias de la maternidad, las jóvenes pierden actividades que están relacionadas con la libertad y con la etapa de la juventud, como el estudio, dejar el hogar familiar u otras. Las renuncias que más están presentes en el discurso de las jóvenes son aquellas que tienen que ver con el esparcimiento y diversión, y se manifiestan en la no posibilidad o limitación de asistir a fiestas: "Tus hijos son una gran bendición y Dios por algo los manda, pero sí dices 'Ay, híjole, el baile ya está lleno y el niño no se duerme' [risa]. Y, o sea, te dice tu mamá el típico 'te lo dije, te lo dije, podrías andar de baile en baile' y uno dice 'no le hace, estoy con m'hijo', pero uno muy en el fondo sí tiene ganas de salir" (Jessica, 16 años).

En el último extracto del discurso de Jessica se puede observar cómo se presenta mayor contradicción entre la compensación de la maternidad y la renuncia a estos espacios de diversión. Parece ser que las fiestas son esos momentos en que las jóvenes recuerdan su libertad perdida y son capaces de renegar del rol de madre para nuevamente autocensurarse con discursos de compensación y responsabilidad asumida. Por otra parte, las jóvenes que abandonaron la educación formal a partir del embarazo parecen tener más presente la pérdida de las relaciones sociales que la continuidad académica, pues en su mayoría retomaron los estudios, al menos hasta el nivel que cursaban cuando quedaron embarazadas.

\section{TRASCENDENCIA}

En las cartas asociativas se presentaron 30 asociaciones de la maternidad con los términos vida y futuro relacionados, por lo que esta categoría remite a la capacidad de dar vida como una forma de trascendencia. Sin embargo, esta significación de la maternidad no se hizo evidente en las entrevistas. En un análisis de correspondencia realizado entre categorías, puede observarse que la trascendencia se ubica de manera cercana a las exigencias y es probable que sean las exigencias que rápidamente se asumen, las que toman un papel más central y relevante en los significados de las madres jóvenes, dejando en un lugar lejano la trascendencia como significación posible de la 
maternidad. Por esta razón, se asume un papel periférico de la trascendencia en la significación de la maternidad en las jóvenes.

\section{PROCESOS/CORPORALIDAD}

Se conforma de elementos asociados a la salud de la madre y/o el hijo y a los procesos de corporalidad que implican el embarazo, parto y posparto, incluyendo conceptos como hospital, cesárea, parto, lactancia, entre otros. Esta categoría tuvo una frecuencia de aparición en las cartas asociativas de 50. A pesar de la alta frecuencia de aparición en las cartas asociativas, en el análisis de correspondencia no se encuentra cercana a las otras categorías. En las entrevistas quedó de manifiesto esta postura distante de la categoría, pues solamente se habló del tema cuando hubo un problema específico de salud o si se asociaba a contenidos de relaciones sociales.

En cuanto a los procesos corporales, al comparar la maternidad temprana con la maternidad tardía, dos de las madres destacan su ventaja corporal sobre las madres mayores. Efectivamente, existen ventajas físicas al comparar a las madres jóvenes con las de mayor edad y parece ser que esto, más el hecho de no haber percibido dificultades de salud en el embarazo, parto o lactancia, permite que las jóvenes tengan como contenido periférico de la representación social o significación de la maternidad a los procesos de corporalidad sin que sean integrados como problemáticos o centrales.

\section{TEMOR/SUFRIMIENTO}

En esta categoría han sido agrupadas asociaciones como miedo, dolor, tristeza y fracaso, entre otras, que dan un significado a la categoría de sentimientos reconocidos socialmente con una carga emotiva negativa y que guardan relación con las demandas de la crianza y cuidado de los hijos, las implicaciones del nuevo rol y la renuncia a actividades que realizan otras jóvenes que no son madres o ellas mismas antes del embarazo. Estos sentimientos se mencionan de manera menos frecuente que los sentimientos con carga emotiva positiva que quedan ejemplificados en la categoría de "compensación". Aun y cuando son menos frecuentes, las jóvenes entrevistadas expresan esa

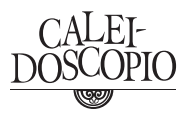


ambivalencia en la vivencia de la maternidad en varias de sus intervenciones:

[...] me siento lista. Al principio no. O sea, los primeros meses fue un derroche de emociones, de cuestiones, de temores. O sea, tenía mucho miedo, tenía muchas dudas, tenía inseguridad, pero poco a poco te das cuenta de que sí puedes, [... | como emoción-amor-felicidad-alegría |risas], y otra en contraparte también es miedo. Miedo a que hagas un mal papel, a no saber qué hacer si se presenta una situación. Y con situaciones me inclinaría, por ejemplo, con preocupación. Entonces emociones sería miedo, preocupación, amor, felicidad, alegría, etc. Y también melancolía; podría decir melancolía, sí, porque pues dejaste atrás toda una vida muy rápido. Igual y la extraño (Ana, 19 años).

Como se puede ver en el extracto de Ana, los sentimientos con carga emotiva negativa están íntimamente relacionados con las exigencias de la maternidad, pero también, en gran medida, con las renuncias, lo cual se aprecia en el discurso de las otras jóvenes entrevistadas. Tanto en las entrevistas como en las cartas asociativas, las jóvenes madres refieren pocos sentimientos o adjetivos negativos como contenidos de la significación de la maternidad. Esto difiere de aquellas representaciones sociales que otros, en su mayoría adultos, tenemos de la maternidad temprana como una situación en que la joven vive culpa, frustración y arrepentimiento.

\section{RETOS}

Se refiere a la significación de la maternidad por parte de la joven en términos de una perspectiva de futuro o plan de vida, tales como embarazo, estudio o trabajo, ya sea a corto, mediano o largo plazo. Estas asociaciones se presentaron con una frecuencia de 19. En el caso de las entrevistadas, las jóvenes hablan de este tema desde una pregunta expresa de la entrevistadora. Al respecto, todas las entrevistadas mencionan la continuidad educativa como un reto que con la maternidad se vislumbra difícil, pero posible. Para algunas, el hijo se convierte en motor para retomar el plan de continuar estudiando. Las dos entrevistadas que en este momento se encuentran estudiando una licenciatura ya no hablan de la educación como un reto a futuro, sino del trabajo. 
La diferencia entre las entrevistadas es que aunque todas hablan de retos a futuro, parece que solamente algunas están comenzando a poner en marcha dicho plan. Se cree que la diferencia entre un plan y una aspiración puede encontrarse en el contexto de las jóvenes, ya que para algunas sus retos son una posibilidad alcanzable, mientras que para otras puede ser un reto que no encuentra las oportunidades para pasar de la aspiración a la ejecución.

\section{DISCUSIÓN}

Con base en los resultados, se puede afirmar que los actores sociales cercanos a las madres jóvenes contribuyen positiva o negativamente en la significación que le dan a la maternidad. Familia, amigos y pareja pueden ofrecer apoyo o rechazo a la joven durante los primeros años de maternidad, lo que se constituye como un factor que puede predecir significaciones de arrepentimiento, deseo de autonomía, amor por los hijos o reparación (Llanes, 2014). Es por esta razón que la discusión comienza en la asociación (binomio) entre el apoyo social y otros contenidos de la representación social de la maternidad para las jóvenes entrevistadas.

Es en los gastos en donde se puede encontrar el nexo más fuerte de relación entre el apoyo social que proporciona el contexto a la joven y la forma en que ésta puede responder a las exigencias de la maternidad. Por esta razón, las jóvenes transitan entre ser mantenidas económicamente por los otros en el inicio de la maternidad y buscar más adelante la autonomía económica, tal como lo comienzan a hacer tres de las entrevistadas (Ana, Mayra y Blanca).

Dentro de los hallazgos de Llanes (2014) y Climent (2003, 2012; Climent, Arias y Spurio, 2000), se encuentra que la relación con los padres es un factor asociado a la ocurrencia o no del embarazo adolescente, pero esta relación incide, sobre todo, en la forma en que la joven puede significar la maternidad una vez que se enfrenta a las exigencias de ésta, ya sea renegando o asumiendo y respondiendo a las exigencias; respuesta cuya conflictividad -nosotros agregamos- puede reducirse sólo si la joven cuenta con el apoyo de una red social, ya sea a nivel económico o a través de la colaboración instrumental en tareas de cuidado y educación del hijo, o bien, dando

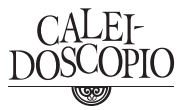


consejos e información sobre cómo implementar patrones de crianza y cuidado.

La asociación entre la compensación y la red de apoyo social de la joven se manifiesta al atribuir una explicación de causalidad al cambio, generalmente en beneficio de la joven, en la relación con padres, hermanos y pareja, tal como lo expresan las jóvenes entrevistadas con enunciados como "ahora que está el niño hemos mejorado nuestra relación entre ellos [refiriéndose a sus padres] y yo" (Diana, 19 años); o "eso [el embarazo y nacimiento del hijo] unió a mi familia" (Ana, 19 años).

El contenido de compensación está presente en todas las entrevistadas como parte central de la representación social, y la manera en que se presenta puede variar dependiendo de las relaciones que se establecen con los otros. El papel que juega la pareja es importante para las jóvenes, ya que, a diferencia de la familia de origen, no siempre se presenta como un apoyo social. Esto guarda relación con el hallazgo de Maldonado y Micolta (1999) respecto a la diferencia de roles de género ante el embarazo adolescente: las jóvenes se convierten en adultas y asumen la maternidad en el marco de unas exigencias sociales claras, mientras que, con la venia de la cultura, los jóvenes pueden seguir siendo adolescentes y no responsabilizarse de la paternidad si así lo desean.

Respecto a la relación de pareja, las jóvenes esperan el apoyo social, pero también esperan que sea cubierta su necesidad de amor romántico. Cuando las jóvenes encuentran en su pareja la necesidad de apoyo resuelta, la compensación gira en torno al hijo como fuente de dicha unión y mejora en la relación de pareja. Por otra parte, cuando la pareja abandona a la joven o incluso se presenta una situación de violencia, entonces la compensación de la maternidad se presenta poniendo en el centro al hijo como motivador para "salir adelante".

En conclusión, la compensación que la maternidad puede tener para las jóvenes adopta las formas de motivación, generación de sentimientos positivos y cambio de hábitos característicos de la madurez. Las diferentes formas positivas que puede tomar la maternidad están estrechamente relacionadas con el apoyo que ofrecen la familia y la pareja, principalmente, tal vez porque, tal como lo manifiestan las jóvenes entrevistadas por Llanes (2014), la imagen de maternidad ideal para las jóvenes sigue una trayectoria en la cual se considera que la 
unión de pareja es relevante, ya sea antes o después del embarazo, y no necesariamente a través del matrimonio.

De tal manera que las representaciones sociales de la maternidad temprana en las jóvenes informantes de esta investigación apuntan a una maternidad idealizada que les permite asumir las exigencias del cuidado y crianza de los hijos, destacando ganancias afectivas como la compensación sobre pérdidas como las renuncias, y relativizando el carácter problemático de la situación, de acuerdo al contexto social de cada una de ellas.

Para llegar a la comprensión de estas representaciones sociales de maternidad temprana, es necesario aludir a la base de su construcción y a su funcionalidad. Los elementos en torno a los cuales las jóvenes construyen su particular visión, significación o representación social de la maternidad se encuentran en la relación que establecen con su red social de apoyo, pero también en la relación con el hijo. Así, tenemos que la red social de apoyo de las jóvenes, vista en términos de clase social y grupo de pertenencia, media sobre el acceso a la información sobre cuidado y crianza de los hijos, incluyendo la imagen de género del ser mujer y el papel de la maternidad en esta imagen. Dentro de esta información se incluyen también los estereotipos de la juventud y adultez, así como las tareas propias de cada etapa dentro del contexto sociocultural de las jóvenes.

La red social de apoyo está constituida principalmente por los padres de la joven, destacando el papel de la madre como proveedora de información y de apoyo instrumental en el cuidado de los hijos. El padre de la joven funge como proveedor o apoyo económico para su hija y su nieto. Por esta razón, los antecedentes educativos, económicos y familiares de la familia de origen sitúan a la joven en una clase social que le da acceso a determinada información, le inculca ciertos estereotipos y aspiraciones, y establece ciertos alcances y límites al enfrentar retos y trazar planes de vida. Estas diferencias de contexto se manifiestan específicamente en diferentes maneras de ser madre joven, sea como única actividad o además de otros posibles planes de vida.

Por otra parte, la relación íntima, cercana y de correspondencia mutua que establecen las jóvenes con sus hijos se convierte en el proveedor de contenidos afectivos positivos en torno a la maternidad, lo que se traduce en la reproducción de una idealización de lo maternal, donde las ganancias emotivas permiten responder de manera

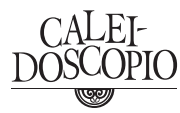


comprometida, asumiendo las exigencias del cuidado y crianza de los hijos, vista por las jóvenes como asumir la responsabilidad.

Las funciones de las representaciones sociales que las jóvenes tienen respecto a la maternidad son, por una parte, las de generar esquemas de conocimiento que posibiliten la integración de información nueva. En este caso, los esquemas tienen que ver con la respuesta a las demandas del cuidado y crianza de los hijos, así como con la información preexistente sobre las exigencias o expectativas de las mujeres adolescentes. Por otra parte, las representaciones sociales son un referente para la identidad grupal como madres y como jóvenes. Así, las jóvenes definen su identidad grupal como madres que responden a las exigencias y como jóvenes que renuncian a actividades culturalmente consideradas propias de la juventud. Otra función de las representaciones sociales de la maternidad es la de orientación, lo que permite a las jóvenes no solamente dar sentido a su comportamiento como madres y jóvenes, sino también conformar expectativas que se materializan en retos. Por último, las representaciones sociales de la maternidad de cada joven son referentes para justificar su actuación, como ocurre cuando las jóvenes narran los sentimientos negativos generados a partir de renuncias que hicieron a espacios de libertad ante la exigencia de la maternidad, para añadir luego los sentimientos positivos atribuidos al nacimiento y relación con el hijo.

\section{CONCLUSIONES}

La visión comprensiva de la maternidad temprana que hemos presentado aporta elementos para poder incidir en el fenómeno a través de la generación de políticas públicas, encaminadas a promover el desarrollo y oportunidades viables para las jóvenes en general, que les permita decidir la maternidad como un plan de vida entre o además de otros posibles; asimismo, políticas públicas que permitan el sano desarrollo de las madres jóvenes y sus hijos como partícipes de la sociedad. Desde la sociedad civil, nuestro estudio apoya el cuestionamiento de la visión adultocéntrica de la juventud, lo que nos lleva a revisar las socializaciones de género que perpetúan la idealización de la maternidad y, a la vez, cuestionan a quienes son madres jóvenes por considerarlas incapaces para tal labor. Así como se han ido integrando 
a las formas de ser madre en nuestra sociedad actual, elementos como el parto humanizado, la lactancia o el porteo como símbolos de un retorno a lo natural de la maternidad, bien podría integrarse la maternidad en la juventud como una opción válida y deseable.

Con ello no se pretendería promover que las jóvenes se embaracen, anteponiendo la maternidad a otros planes de vida posibles, como reproducción del patriarcado y en oposición frontal a algunas posturas feministas que hablan de postergar o descartar la maternidad. Más bien se trata de considerar una posición feminista que Saletti (2008) identifica como reconstructora de la maternidad como fuente de placer, conocimiento y poder femeninos, la cual está centrada en rescatar y empoderar a las mujeres a través del cuerpo y la maternidad. Esta posición bien podría revalorizar la posibilidad de ser madre en la juventud, no en vez de o después de, sino además de cualquier proyecto de vida posible para las mujeres. Se abre así la posibilidad de responder de manera positiva a la pregunta que se hace Climent (2003) acerca de si acaso la maternidad en la juventud debería ser una pauta social a respetar.

Si la maternidad temprana es un fenómeno que puede revalorizarse $\mathrm{o}$, al menos, respetarse y se opta por centrar los esfuerzos de la prevención del embarazo adolescente en la promoción de relaciones de pareja democráticas, en las cuales las jóvenes puedan decidir de manera informada si desean ser madres y cuándo hacerlo, entonces, una vez que la joven decida ser madre o, de facto, esté en camino de serlo, se debe apoyar socialmente su plenitud en la maternidad como uno de los tantos posibles planes de vida que existen para las mujeres. Para saber si la maternidad temprana es una pauta social, es necesario ahondar en las investigaciones sobre este fenómeno pero de una manera comprensiva y respetuosa, reconociendo que las jóvenes madres se saben tan eficaces como cualquier otra. En este tenor, se reconoce que hay tópicos no tratados en esta investigación, como la violencia de género, pobreza, aborto o educación sexual. Nuestro aporte teórico-metodológico sobre el tema y la posibilidad de dar voz a las madres jóvenes puede ser una guía para posteriores investigaciones.

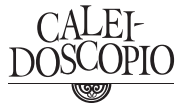




\section{REFERENCIAS}

Abric, J. C. (2001). Prácticas sociales y representaciones. México: Ediciones Coyoacán.

Álvaro, J. L. y Fernández, B. (2006). Representaciones sociales de la mujer. Athenea Digital. Revista de pensamiento e investigación social, 1(9), 65-77. DOI: 10.5565/rev/athenead/v1n9.261.

Castorina, J. A. (2003). Representaciones sociales. Problemas teóricos y conocimientos infantiles. Barcelona: Gedisa.

Climent, G. (2003). La maternidad adolescente, una expresión de la cuestión social. El interjuego entre la exclusión social, la construcción de la subjetividad y las políticas públicas. Revista Argentina de Sociología, 1(1), 77-93. Recuperado de: http://www.redalyc.org/articulo. oa? $\mathrm{id}=26900106$.

Climent, G. (2009). Representaciones sociales sobre el embarazo y el aborto en la adolescencia: perspectivas de las adolescentes embarazadas. Cuadernos de la Facultad de Humanidades y Ciencias Sociales. Universidad Nacional de Jujuy, (37), 221-242. Recuperado de: http:// www.redalyc.org/articulo.oa?id=18516803009.

Climent, G. (2012). Maternidades adolescentes heterogéneas. El impacto de las relaciones familiares y los estudios educativos parentales. Comunicación presentada en el V Congreso de la asociación Latinoamericana de Población. 23-26 de octubre, Montevideo (Uruguay). Recuperado de: http://www.alapop.org/Congreso2012/ DOCSFINAIS_PDF/ALAP_2012_FINAL447.pdf.

Climent, G., Arias, D. y Spurio, C. (2000). Maternidad adolescente: un camino hacia la marginación. Cuadernos médicos sociales, 77, 81-101.

Jodelet, D. (1985). La representación social: fenómenos, concepto y teoría. En S. Moscovici (Ed.). Psicología Social II. Pensamiento y vida social. Psicología social y problemas sociales (pp. 469-494). Barcelona: Paidós.

Lamus, D. (1999). Representaciones Sociales de Maternidad y Paternidad en Cinco Ciudades Colombianas. Reflexión Política, 1(2). DOI: $10.29375 / 01240781.887$.

Llanes, N. (2014). "Estar en la edad". Re-significaciones de la maternidad adolescente en un contexto de alta inmigración: el caso de mujeres residentes en Tijuana (Tesis doctoral). Baja California: El Colegio de la Frontera Norte. Recuperado de: http://www.colef.mx/posgrado/wp-content/ uploads/2014/11/Tesis-Llanes-Diaz.pdf. 
Maldonado, M. C. y Micolta, C. (1999). Los procesos vitales cruzados en padres y madres adolescentes. Investigación y Desarrollo, (9). Recuperado de: http://rcientificas.uninorte.edu.co/index.php/investigacion/article/view/2692/1804.

Moscovici, S. y Hewstone, M. (1986). De la ciencia al sentido común. En S. Moscovici (Ed.). Psicología Social II. Pensamiento y vida social. Psicología social y problemas sociales (pp. 679-710). Barcelona: Paidós.

Navarro, P. y Díaz, C. (1995). Análisis de contenido. En J. M. Delgado y J. Gutiérrez (Eds.). Métodos y técnicas cualitativas de investigación en ciencias sociales. Madrid: Síntesis.

Ortiz, D. y Maza, O. (2010). Madres solas y adolescentes en Aguascalientes. Aportaciones desde la perspectiva de género para el diseño de políticas públicas. Aguascalientes, México: Instituto Aguascalentense de las Mujeres. Recuperado de: http://cedoc.inmujeres.gob.mx/ftpg/ Aguascalientes/agsmeta8.pdf.

Perera, M. P. (1999). A propósito de las representaciones sociales. Apuntes teóricos, trayectoria y actualidad. La Habana: Centro de Investigación en Estudios Psicológicos y Sociológicos. Recuperado de: http://biblioteca.clacso.edu.ar/Cuba/cips/201306281 10808/Perera_perez_repr_ sociales.pdf.

Román, R. (2000). Del primer vals al primer bebé. Vivencias del embarazo en las jóvenes. México: CIEUV-IMJ. Recuperado de: https://www.researchgate. net/publication/31704152_Del_primer_vals_al_primer_bebe_vivencias_del_embarazo_en_las_jovenes_R_Roman_Perez_presen_de_ LI_Sanchez_Gomez.

Saletti, L. (2008). Propuestas teóricas feministas en relación al concepto de maternidad. CLEPSYDRA, 7, 169-183.

Schwarz, P. (2005). Influencia de las representaciones sociales de la maternidad en la construcción de identidad femenina en mujeres jóvenes de clase media urbana. Comunicación presentada en las III Jornadas de Jóvenes Investigadores. 29 y 30 de septiembre, Buenos Aires (Argentina). Recuperado de: http://webiigg.sociales.uba.ar/ iigg/jovenes_investigadores/3JornadasJovenes/ Templates/Eje\%20 identidad-alteridad/Schwarz-identidad.pdf.

Stern, C. (2012). El "problema" del embarazo adolescente: contribuciones a un debate. México, D.F.: El Colegio de México. 
Bélgica María Romero de Loera (belgicaromero@gmail.com) es doctora en Estudios Socioculturales (UAA) con la tesis Representaciones sociales de la maternidad en madres jóvenes; maestra en Tecnología Educativa (ITESM) y licenciada en Psicología (UAA). Actualmente participa como parte del cuerpo docente del Doctorado en Ciencias de la Educación en la Universidad Cuauhtémoc de Aguascalientes, en la Maestría en Educación de la Universidad Tecnológica en Línea y como tutora académica en la Universidad Virtual del Estado de Guanajuato (ORCID 0000-0002-4029-7377).

\section{CÓMO CITAR ESTE ARTÍCULO}

Romero, B. M. (2018). Binomios y dicotomías en las representaciones sociales de la maternidad en madres adolescentes. Caleidoscopio. Revista Semestral de Ciencias Sociales y Humanidades, 22(39). DOI:10.33064/39cr$\operatorname{scsh} 14240$ 